\title{
Outcomes after operative treatment of patella fractures
}

\author{
V.Kh. Khidzhazin ${ }^{1}$, E.I. Solod ${ }^{1,2}$, M.A. Abdulkhabirov ${ }^{1}$
}

${ }^{1}$ Peoples' Friendship University of Russia, Moscow, Russian Federation,

${ }^{2}$ National Medical Research Center of Traumatology and Orthopedics n.a. N.N. Priorov, Moscow, Russian Federation

\begin{abstract}
Introduction Patella fractures account for one percent of all fractures. Arthritis, non-union and other complications which affect the function of the lower limb may develop in the long term. The aim was to develop the most optimal osteosynthesis techniques for patella fractures. Methods We analyzed long-term treatment results of 78 patients with patella fractures treated from 2017 to 2018 using the KOOS (The Knee Injury and Osteoarthritis Outcome Score) scale, clinical examinations and knee joint X-rays. Based on the methods of surgical treatment for patella fractures, we divided the patients into 5 groups: Weber tension band wiring for two-fragment fractures; cross-like osteosynthesis and other methods of patella osteosynthesis using K-wires and wire loops for multifragmental fractures of the patella; combined osteosynthesis (tension band wiring with an additional screw or cerclage around patella); partial patellectomy and the inferior pole osteosynthesis. Results A comparative analysis of patients' groups after surgical treatment of patella fractures using the KOOS scale showed the best results after Weber tension band wiring for transversal fractures (72 \%). The results of patients with multifragmental fractures of the patella using K-wires and wire loops were positive in $64 \%$; after the restoration of the inferior pole in $68 \%$, after partial patellectomy in $57 \%$. Conclusion For transversal fractures of the patella, tension band wiring is the method of choice. With multifragmental patella fractures, good results are achieved by cross-like osteosynthesis and other methods using K-wires and wire bands. The use of patellectomy followed by wire loop locking should be avoided for all types of patella fractures. In fractures of the inferior pole of the patella, it is preferable to fix it to the main patella fragment.
\end{abstract}

Keywords: patella fractures, osteosynthesis, KOOS, outcomes

\section{INTRODUCTION}

Patella fractures account for about $1 \%$ of all skeletal fractures [1-5]. Patients with patella fractures without displacement are usually treated conservatively with plaster cast immobilization of the lower extremity from four to six weeks $[4,6]$. Surgical treatment methods are applied in patients with patella fractures when fragments are shifted from one to $4 \mathrm{~mm}$ and articular surface incongruency of more than $2 \mathrm{~mm}[4,5,7]$. The Weber's method of patella osteosynthesis for twofragment patella fractures using two wires and an eightshaped wire loop is the most used technique $[7,8]$. However, there is no single approach to the treatment of patients with multifragmental patella fractures among the specialists. Taylor B. [9] and Lorich D. [10] use plates of different designs for osteosynthesis of complex fractures. Joseph, J. et al. apply partial patellectomy [11].
Various complications may be observed after surgical treatment of patients with patella fractures (wire migration, infection, knee joint contractures and patellofemoral arthritis) [2-7]. LeBrun Cristopher T. et al. (2012) published long-term results of 40 patients after patella fractures who underwent not only Weber's osteosynthesis but also osteosynthesis with cannulated screws, longitudinal anterior banding with cerclage and even partial patellectomy. However, the authors do not analyze the outcomes after these operations [12]. Moreover, there is no consensus on the treatment of complex fractures of the patella, and traumatologists also use a wide variety of methods for osteosynthesis of the patella in their practice. In connection with the foregoing, the study of long-term results in patients after patella fractures is of particular relevance.

\section{MATERIAL AND METHODS}

We followed 78 patients with patella fractures who underwent surgical treatment from 2014 to 2018. All patients signed informed consents for participating in the study in accordance with the WHO Declaration of Helsinki on ethical principles for medical research involving human subjects (2013). The study was approved by the ethics committee of the institution. Thirty-eight patients with two-fragment fractures underwent classical osteosynthesis with two wires and an 8-shaped tension band according to Weber; six patients with tri-fragment fractures were treated with three wires and two tension bands according to 
the diagram shown in Fig. 1; two patients with fourfragment fractures had "cross-like" osteosynthesis with four wires and two tension bands (Fig. 2) (Patent invention No. 2615665 dated April 06, 2017 "Method for osteosynthesis of multifragmental patella fractures" by V. Khidzhazin, N.V. Zagorodni, M.A. Abdulkhabirov. Six wires with three tension bands were used for osteosynthesis in one patient with a multifragmented (more than 6 fragments) fracture of the patella in order to preserve all the fragments. In case of fractures of the inferior pole of the patella, we also tried to preserve it. For this purpose, the distal fragment was fixed with a double stitch to suture the inferior pole to the main fragment by reinforcing the ligament of the patella on both sides (Fig. 3). Moreover, at a follow-up appointment, we retrospectively observed seven patients who underwent combined osteosynthesis of the patella (Weber's osteosynthesis with additional fixation of the fragments with a screw or cerclage around the patella), and 21 patients who underwent partial patellectomy.

Based on the facts that various methods of surgical treatment for patella fractures were used and depended on the fracture type as well as due to a small number of patients in some groups and for facilitating the statistical analysis, we divided the patients into the following groups: Weber tension band wiring for two-fragment fractures; cross-like osteosynthesis and
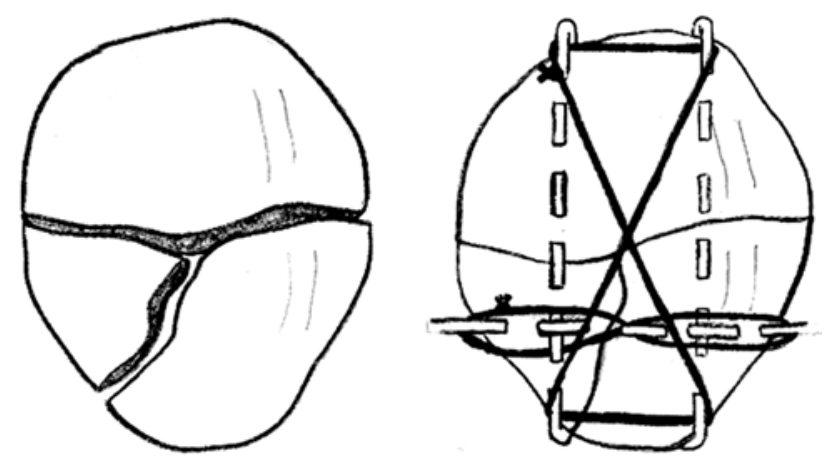

Fig. 1 Diagram of osteosynthesis of the patella with three wires and two tension bands

other similar methods of patella osteosynthesis using $\mathrm{K}$-wires and wire bands for multifragmental fractures of the patella; combined osteosynthesis (tension band wiring with an additional screw or cerclage around patella); partial patellectomy in comminution of the distal patellar pole; inferior pole osteosynthesis with double suture and partial reinforcement of the patellar ligament from both sides.

The treatment of patella fractures was evaluated with the KOOS score. Each KOOS question suggests five answers in points, from 0 to 4 (no - 0 , mild - 1 , moderate -2 , strong -3 , severe -4 ). For each subscale, the total score was calculated. The total count of points was made according to special formulas to obtain a point score from 0 to 100 points. Moreover, 100 points corresponded to absence of symptoms, and 0 points indicated the severity of all symptoms.

The results of surgical treatment were evaluated between eight months and three years after surgery; the average follow-up time was one year and ten months.

The reliability of the results was determined automatically using the program for processing statistical data (R-language), by entering the average values of the results in each group.

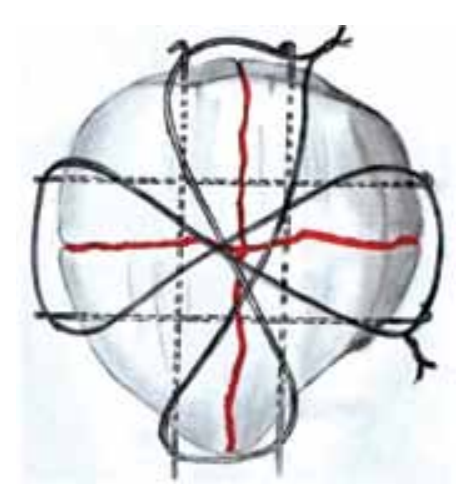

Fig. 2 Diagram of cross-like osteosynthesis

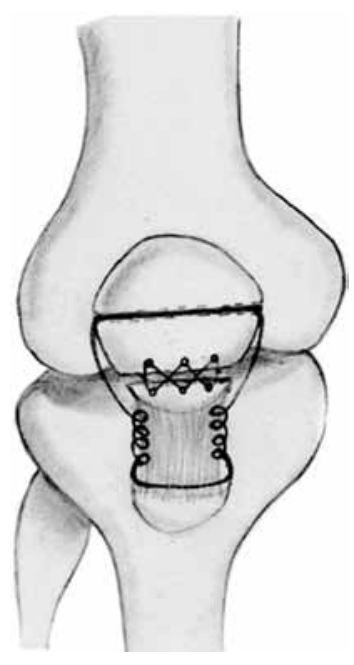

Fig. 3 Diagram of fixation of the inferior pole with sutures and partial reinforcement of the patella ligament from both sides

\section{RESULTS}

Metal elements were removed in 40 (51\%) out of 78 patients at the time of follow-ups (Table 1 ).

The Table 1 shows the ratio of patients' referrals for removal of metal patella fixators in Weber's osteosynthesis groups, "cruciform" osteosynthesis, and other methods of osteosynthesis with wiring and combined osteosynthesis was approximately equal (29 $\%, 33 \%, 29 \%$, respectively). If the inferior pole of the patella was fixed to its main fragment by transosseous suture with partial reinforcement of the patellar ligament on both sides there was no need for repeated surgical interventions to remove them due to the absence of metal 
fixators, which is a great advantage in the treatment of patients with this type of fracture. After partial patellectomy with the application of a locking wire loop, 19 out of 21 patients (90\%) applied for its removal due to breakage and separation. There were technical difficulties were noted during operations to remove the locking wire loop, especially when it was fragmented.

KOOS questionnaires showed the following results after surgical treatment (average values) for subscales: symptoms $-72.2 \%$, pain $-76.4 \%$, daily activity $-60.2 \%$, quality of life $-64.5 \%$, sports and recreation $-59.7 \%$. The rating for each group of patients is shown in Table 2.

A comparative analysis of patients after surgical treatment of patella fractures, as seen from the Table, showed the best results after Weber's osteosynthesis for two-fragment patella fractures, which is consistent with the published data. The treatment results of multifragmental patella fractures were inferior in their effectiveness due to severity of the fractures. The results of treatment of patients with complex fractures of the patella with cruciform osteosynthesis and other similar methods of osteosynthesis with tension band wiring (68\%), exceeded the results of treatment of patients after patellectomy $(51 \%)(p=0.021)$.

To assess the functional result of treatment, we supplemented the scale by examining the range of active and passive movements not only in the injured knee joint, but also in the healthy one. Evaluation results showed mild flexion restrictions in 4 of 21 patients (within 90-130 degrees), two patients noted discomfort with complete flexion in the knee joint.

Moreover, we considered our patients' own assessment of the results of their treatment as good, satisfactory and poor. Good results were expressed by $56 \%$ of patients, satisfactory by $30 \%, 12 \%$ of patients expected a better result, and $2 \%$ of patients did not give a clear answer.

Table 1

The number of operations to remove metal after surgical treatment of patients with patella fractures

\begin{tabular}{|l|c|c|c|}
\hline Type of intervention & $\begin{array}{c}\text { Number of patients } \\
(\mathrm{n})\end{array}$ & $\begin{array}{c}\text { Removal of fixation } \\
\text { elements }\end{array}$ & \% from the total \\
\hline Weber's osteosynthesis & 38 & 11 & 29 \\
\hline $\begin{array}{l}\text { Crusiform osteosynthesis and other methods } \\
\text { with the use of wires and tension bands }\end{array}$ & 9 & 3 & 33 \\
\hline $\begin{array}{l}\text { Combined methods (Weber + screw or } \\
\text { cerclage) }\end{array}$ & 7 & 2 & 29 \\
\hline Partial patelloectomy & 21 & 19 & 90 \\
\hline
\end{tabular}

Table 2

KOOS evaluation in each group of patients after surgical treatment

\begin{tabular}{|c|c|c|c|c|c|c|}
\hline Type of intervention and number of patients & $\begin{array}{c}\text { Symptomatic } \\
(\%)\end{array}$ & $\begin{array}{c}\text { Pain } \\
(\%)\end{array}$ & \begin{tabular}{|c|}
$\begin{array}{c}\text { Activities } \\
\text { of daily life } \\
(\%)\end{array}$ \\
\end{tabular} & $\begin{array}{l}\text { Quality } \\
\text { of life } \\
(\%)\end{array}$ & $\begin{array}{c}\text { Sport and } \\
\text { recreation } \\
(\%)\end{array}$ & Total (\%) \\
\hline Weber's osteosynthesis $(\mathrm{n}=38)$ & 76 & 80 & 66 & 74 & 64 & 72 \\
\hline $\begin{array}{l}\text { Crusiform osteosynthesis and other methods } \\
\text { with the use of wires and tension bands }(n=9)\end{array}$ & 75 & 73 & 63 & 68 & 61 & 68 \\
\hline Combined methods $(n=7)$ & 70 & 72 & 56 & 62 & 60 & 64 \\
\hline Osteosynthesis of the inferior pole $(n=3)$ & 87 & 76 & 55 & 62 & 55 & 67 \\
\hline Partial patellectomy $(n=21)$ & 47 & 55 & 51 & 50 & 52 & 51 \\
\hline
\end{tabular}

\section{DISCUSSION}

Despite the fact that the KOOS system has been recognized by many colleagues, we opine that there are some gaps in its use for assessing the condition of patients after osteosynthesis of the patella as a result of studying long-term results.

The KOOS score system does not consider the condition of the knee joint before the patella fracture, as well as the age of patients and concomitant diseases of both the osteoarticular system and other organs and systems that impede the normal functioning of the knee joint, which undoubtedly have an impact on the outcome of treatment. Moreover, the "activity of daily life" and "sport and recreation" subscales are not very suitable for elderly patients, since they are mainly not involved in sports and are not very active.

In connection with the above, we supplemented the 
methods for assessing the results of patients with patella fractures with X-ray studies to identify arthritic changes in the knee joint if patients complained of pain.

Based on the scientific literature and our experience in treating patients, we concluded that osteosynthesis according to Weber remains the optimal method for two-fragment patella fracture fixation. The integrity of the patella is preserved and it is possible to avoid partial or total patellectomy even in multifragment fractures. Gewinner et al. believe that the implementation of partial patellectomy reduces the space between the patella and the femur, resulting in an increase in their contact pressure, development of arthritis and weakening the strength of the quadriceps [3]. In comminuted fractures of the inferior pole of the patella, Kastelec M., Veselko M. showed the advantage of using "basket" plates compared to patellectomy. In addition, the authors noted a significant frequency of low patella (patella baja) with this technique as compared with osteosynthesis [13]. Oh H. and Song H. also tried to avoid patellectomy and used vertical wiring for fractures of the inferior pole. However, they did not fix the patella with an additional wire loop [14, 15].

Despite the above facts, J. Joseph and M. Manoj continue practicing partial patellectomy for comminuted fractures of the patella and recommend the patella fixation with a locking wire loop [11]. Based on the postulate that there is nothing superfluous in the human skeleton, and given the good blood supply to the patella, we consider it necessary and important to preserve all its fragments, fixing them with three or more wires tensioned into two or three wire bands. We also recommend not to remove the inferior pole if torn, but to fix the torn fragment to the main one with transosseous suture with additional reinforcement of the patellar ligament on both sides and avoiding fixing the patella with a locked wire loop. This technique showed good results and allowed to achieve stability without removing the patella or fixing it with a wire loop.

\section{CONCLUSIONS}

The choice of methods for treating multifragment patella fractures remains debatable. Evaluation of the treatment results in patients with patella fractures according to the KOOS showed different values depending on the type of injury, fixation technique and age. "Cross-shaped" osteosynthesis of multifragment patella fractures and osteosynthesis using three wires and two tension bands for three-fragment fractures are the methods of choice. Patellectomy with additional fixation by a locking wire loop should be avoided due to the need to remove it after 6 to 8 weeks, as well as to eliminate complications such as low patella position (patella baja). For detachments of the lower pole, its fixation seems optimal with transosseous suture and partial reinforcement of the patellar ligament on both sides.

Conflict of interest: not declared.

Source of funding: the study was conducted without sponsorship.

\section{REFERENCES}

1. Jarraya M., Diaz L.E., Arndt W.F., Roemer F.W., Guermazi A. Imaging of patellar fractures. Insights Imaging, 2017, vol. 8, no. 1, pp. 49-57. DOI: 10.1007/s13244-016-0535-0.

2. Neumann M.V., Niemeyer P., Südkamp N.P., Strohm P.C. Patellar fractures - a review of classification, genesis and evaluation of treatment. Acta Chir. Orthop. Traumatol. Cech., 2014, vol. 81, no. 5, pp. 303-312.

3. Gwinner C., Märdian S., Schwabe P., Schaser K.D., Krapohl B.D., Jung T.M. Current concepts review: Fractures of the patella. GMS Interdiscip. Plast. Reconstr. Surg. DGPW, 2016, vol. 5, Doc01. DOI: 10.3205/iprs000080.

4. Schuett D.J., Hake M.E., Mauffrey C., Hammerberg E.M., Stahel P.F., Hak D.J. Current treatment strategies for patella fractures. Orthopedics, 2015, vol. 38, no. 6, pp. 377-384. DOI: 10.3928/01477447-20150603-05.

5. Melvin J.S., Mehta S. Patellar fractures in adults. J. Am. Acad. Orthop. Surg., 2011, vol. 19, no. 4, pp. 198-207.

6. Bergin P., Lawyer T.J. Patella Fractures. Eltorai A., Eberson C., Daniels A., eds. Orthopedic Surgery Clerkship. Springer, Cham, 2017, pp. 329-333. DOI: 10.1007/978-3-319-52567-9_71.

7. Bui C.N., Learned J.R., Scolaro J.A. Treatment of Patellar Fractures and Injuries to the Extensor Mechanism of the Knee: A Critical Analysis Review. JBJS Rev., 2018, vol. 6, no. 10, pp. e1. DOI: 10.2106/JBJS.RVW.17.00172.

8. Egol K., Howard D., Monroy A., Crespo A., Tejwani N., Davidovitch R. Patella fracture fixation with suture and wire: you reap what you sew. Iowa Orthop. J., 2014, vol. 34, pp. 63-67.

9. Taylor B.C., Mehta S., Castaneda J., French B.G., Blanchard C. Plating of patella fractures: techniques and outcomes. J. Orthop. Trauma, 2014, vol. 28, no. 9, pp. e231-e235. DOI: 10.1097/BOT.0000000000000039.

10.Lorich D.G., Warner S.J., Schottel P.C., Shaffer A.D., Lazaro L.E., Helfet D.L. Multiplanar fixation for patella fractures using a lowprofile mesh plate. J. Orthop. Trauma, 2015, vol. 29, no. 12, pp. e504-e510. DOI: 10.1097/BOT.0000000000000415. 
11.Joseph B.J., Manoj M.K., Jose F.C. A study of functional outcome of patellar fractures treated with partial patellectomy. Indian J. Applied Res., 2018, vol. 8, no. 3. DOI : 10.36106/ijar.

12.LeBrun C.T., Langford J.R., Sagi H.C. Functional outcomes after operatively treated patella fractures. J. Orthop. Trauma, 2012, vol. 26, no. 7, pp. 422-426. DOI: 10.1097/BOT.0b013e318228c1a1.

13.Kastelec M., Veselko M. Inferior patellar pole avulsion fractures: osteosynthesis compared with pole resection. J. Bone Joint Surg. Am., 2004, vol. 86, no. 4, pp. 696-701. DOI: 10.2106/00004623-200404000-00005.

14.Oh H.K., Choo S.K., Kim J.W., Lee M. Internal fixation of displaced inferior pole of the patella fractures using vertical wiring augmented with Krachow suturing. Injury, 2015, vol. 46, no. 12, pp. 2512-2515. DOI: 10.1016/j.injury.2015.09.026.

15.Song H.K., Yoo J.H., Byun Y.S., Yang K.H. Separate vertical wiring for the fixation of comminuted fractures of the inferior pole of the patella. Yonsei Med. J., 2014, vol. 55, no. 3, pp. 785-791. DOI: 10.3349/ymj.2014.55.3.785.

Received: 04.06.2019

\section{Information about the authors:}

1. Valentin Kh. Khidzhazin,

Peoples’ Friendship University of Russia, Moscow, Russian Federation,

Email: hvalentino@mail.ru

2.Eduard I. Solod, M.D., Ph.D.,

Peoples' Friendship University of Russia, Moscow, Russian Federation,

National Medical Research Center of Traumatology and Orthopedics n.a. N.N. Priorov, Moscow, Russian Federation, Email: Doctorsolod@mail.ru

3. Magomed A. Abdulkhabirov, M.D., Ph.D., associate professor, Peoples' Friendship University of Russia, Moscow, Russian Federation, Email: Abdulkhabirov@yandex.ru 